\title{
Notificação imprecisa da ingestão energética entre usuários de Serviços de Promoção à Saúde
}

\author{
Misreporting of energy intake \\ among users of Health Promotion Services
}

Clesiane Honorato Machado ${ }^{1}$

Aline Cristine Souza Lopes ${ }^{1}$

Luana Caroline dos Santos ${ }^{1}$

${ }^{1}$ Escola de Enfermagem, Departamento de Nutrição, Universidade Federal de Minas Gerais. Av. Professor Alfredo Balena 190/324,

Santa Efigênia. 30130-100

Belo Horizonte MG Brasil. clesianehonorato@

gmail.com
Abstract The assessment of dietary intake is important in nutrition science, however the methods used for this purpose may lead to misreporting, thereby skewing the results associated with diet. This study aims to assess the prevalence of misreporting of dietary intake and associated factors. This is a cross-sectional study with users of nine Health Promotion Services - called 'City fitness facilities' - in Belo Horizonte in the State of Minas Gerais. Sociodemographic and economic data were collected together with information on health history and perceptions, dietary habits and nutritional status. The assessment of energy intake was performed using 24-hour dietary recalls and misreporting of energy intake was determined using the McCrory method. Of the 487 users assessed, 82.3\% were female and 50.5\% were elderly. The prevalence of underreporting was $11,9 \%$, being more prevalent among participants with dyslipidemia and very high waist circumference and was less prevalent among adults, people satisfied with their body weight and those with appropriate meal frequency. Overreporting was found in only $0.8 \%$ of the sample. Underreporting was identified as being a problem in this population, thus calling for strategies for improving dietary reporting accuracy.

Key words Energy intake, Diet surveys, Primary Health Care
Resumo A avaliação da ingestão alimentar é importante na ciência da nutrição, no entanto, os métodos utilizados para tal fim podem levar à notificação imprecisa comprometendo os resultados associados à dieta. Assim, este estudo teve como objetivo avaliar a prevalência de notificação imprecisa da ingestão energética e seus fatores associados. Trata-se de um estudo transversal com usuários de nove academias da cidade de Belo Horizonte, Minas Gerais. Coletou-se dados sociodemográficos e econômicos, história e percepção de saúde, hábitos alimentares e estado nutricional. A avaliação da ingestão energética deu-se por Recordatórios Alimentares de 24 horas e a notificação imprecisa pela metodologia de McCrory. Avaliaram-se 487 usuários, $82,3 \%$ do sexo feminino e $50,5 \%$ idosos. A prevalência de subnotificação do consumo alimentar foi de 11,9\%, sendo mais prevalente entre os participantes com dislipidemiase circunferência da cintura muito elevada, e menos prevalente entre os adultos, os satisfeitos com o peso corporal e com fracionamento da dieta igual ou superior a cinco refeições diárias. A supernotificação foi encontrada em 0,8\% da amostra. Identificou-se a subnotificação como um problema nessa população, demandando estratégias para o aprimoramento da notificação dietética.

Palavras-chave Ingestão de energia, Inquéritos sobre dietas, Atenção Primária à Saúde 


\section{Introdução}

A avaliação da ingestão alimentar é de extrema importância na ciência da nutrição, estando, entre as suas diversas aplicações, a identificação de problemas nutricionais a partir do monitoramento da ingestão de alimentos e nutrientes, e a determinação de associações entre a ingestão alimentar e o processo saúde/doença ${ }^{1}$.

Para tal, tornam-se necessários métodos de inquérito alimentar válidos e que forneçam informações confiáveis ${ }^{2}$ que estão susceptíveis ao fenômeno da notificação imprecisa, a qual compreende tanto a super (relato de alimentos que não foram ingeridos e/ou aumento das quantidades) quanto a subnotificação (não relato de todos os alimentos ingeridos e/ou relato em quantidades menores). Essa imprecisão pode comprometer, seriamente, a interpretação dos resultados de pesquisas que relacionam nutrição e saúde, sobretudo quando se refere ao conteúdo energético da dieta, interferindo, consequentemente, na avaliação da ingestão de nutrientes ${ }^{3,4}$.

Essa ocorrência da notificação imprecisa pode ser determinada por métodos que validam a ingestão energética autorreferida ${ }^{3,5}$, propiciando magnitudes diferentes entre os estudos e as populações ${ }^{5}$. Estudo de revisão bibliográfica apontou prevalência de subnotificação da ingestão alimentar variando de $21,5 \%$ a $67 \%$, e de supernotificação, de $1 \%$ a $6 \%$ entre as mulheres e 1,6\% entre homens, com o uso do R24h como método para avaliação do consumo alimentar. Já com a utilização do diário alimentar, a subnotificação variou de $11,9 \%$ a $44 \%$, enquanto a supernotificação, de 3,5\% a 7\% ${ }^{6}$.

Diante da importância dessa avaliação nos estudos para uma melhor qualidade dos dados, a escassez na literatura de trabalhos nacionais que realizem tal investigação, somando que esse fenômeno é altamente influenciado por fatores sociais e culturais, torna-se fundamental estudá -lo em qualquer população na qual se pretenda mensurar o consumo alimentar ${ }^{7}$. Além disso, é importante, ainda, determinar os diferentes fatores associados à notificação imprecisa para elucidar o modo com o qual as características dos sujeitos influenciam no relato de ingestão de alimentos, aumentando, assim, a compreensão deste processo a fim de favorecer a fidedignidade da avaliação e delineamento de intervenções mais apropriadas ${ }^{4}$.

Dessa forma, buscou-se avaliar a prevalência de notificação imprecisa do consumo energético e seus fatores associados entre usuários de Ser- viços de Promoção da Saúde de Belo Horizonte, Minas Gerais.

\section{Métodos}

Trata-se de um estudo transversal realizado entre 2013 e 2014 no contexto das Academias da Saúde, que fazem parte de uma estratégia do projeto de Promoção de Modos de Vida Saudáveis "BH +Saudável”, elaborado pela Secretaria Municipal de Saúde de Belo Horizonte, com o objetivo principal de contribuir para a promoção da saúde e a produção do cuidado e de modos saudáveis de vida da população ${ }^{8,9}$.

Este trabalho foi uma parte do projeto intitulado "Consumo de frutas e hortaliças em serviços de promoção da saúde de Belo Horizonte, Minas Gerais: fatores associados e intervenções nutricionais", desenvolvido em 18 Academias da Saúde, duas em cada região administrativa do município, sendo esse número obtido por meio de processo amostral de forma a se alcançar uma amostra representativa do serviço no município, considerando o Índice de Vulnerabilidade à Saúde, assumindo-se como premissa que as Academias atendam usuários com distintos índices, já que são serviços de saúde com acesso universal, localizados em pontos estratégicos das regionais, garantindo variabilidade amostral ${ }^{9}$.

Para o desfecho do presente estudo realizou-se um cálculo amostral considerando uma população média de 265 usuários no turno matutino por Academia da Saúde de $\mathrm{BH}$ a partir de informações da prefeitura, erro alfa de 5\%, 95\% de confiança e percentual máximo esperado de notificação imprecisa de 29,6\% (27\% de subnotificação e 2,6\% de supernotificação) utilizando-se como método de avaliação do consumo alimentar o R24h ${ }^{6}$, obtendo-se um número estimado de 316 indivíduos ${ }^{10}$. Assim, utilizou-se dados de nove Academias da Cidade, uma em cada região administrativa do município.

O estudo foi realizado com todos os usuários das Academias da Cidade que atendessem aos critérios de inclusão: ter 20 anos ou mais de idade, aceitar participar da pesquisa por meio da assinatura do Termo de Consentimento Livre e Esclarecido e, para fins de análise, declaração de não estar realizando alguma tentativa atual para perda ou ganho de peso em virtude da sua implicação na notificação do consumo alimentar e possível confundimento nas análises ${ }^{11}$. Foram excluídas gestantes, usuários que faltaram a três marcações para a realização das entrevistas e 
aqueles que necessitavam de auxílio para responder o questionário.

Utilizou-se, para a coleta de dados, questionário estruturado pré-codificado e pré-testado, que consta de variáveis sociodemográficas e econômicas (idade - categorizada em adulto e idoso, sexo, profissão, renda per capita, e escolaridade), história e percepção de saúde, consumo alimentar e estado nutricional.

As informações foram coletadas por acadêmicos de graduação em Nutrição e Pós-graduação em Enfermagem e Saúde de uma universidade federal e por nutricionistas, e todos os entrevistadores passaram por treinamento no início do projeto e periodicamente para atualização e esclarecimento de dúvidas que surgiram durante a coleta. Contou-se ainda com um manual elaborado para fins do próprio estudo que continha instruções e padronização para todas as questões do protocolo. Ademais, todos os entrevistadores eram acompanhados por um supervisor responsável e realizava-se contínua análise de consistência das informações obtidas.

Os dados referentes à história e percepção de saúde incluíram morbidades autorreferidas (diabetes mellitus - DM, hipertensão arterial sistêmica - HAS e dislipidemias), morbidade psiquiátrica segundo o tratamento psiquiátrico autorreferido $^{12}$, uso de medicamentos, tabagismo, percepção de saúde (categorizada em muito boa/ boa, razoável e ruim/muito ruim), satisfação com o peso atual, e se alguma vez na vida já receberam orientação de algum profissional de saúde para mudança dos hábitos alimentares.

Quanto ao exercício físico, questionou-se a frequência semanal e o tempo diário dessa prática, para posterior classificação do nível segundo recomendações do Institute of Medicine $(\mathrm{IOM})^{13}$.

Em relação ao consumo alimentar, aplicou-se dois Recordatórios Alimentar de 24h (R24h) presencialmente e um terceiro via telefone para uma subamostra de $25 \%$ dos participantes, ao longo de dias não consecutivos, contemplando todos os dias da semana, distribuídos aleatoriamente entre os indivíduos da amostra. Todos os participantes foram orientados sobre a importância de relatar o real consumo e, para facilitar o processo e minimizar os erros referentes à estimativa do tamanho das porções, utilizou-se, em uma amostra aleatória, um kit de medidas caseiras contendo utensílios diversos comumente usados pela população em estudo.

Os alimentos relatados tiveram suas medidas caseiras transformadas em gramas ou mililitros e o valor energético e o conteúdo de macronutrien- tes, colesterol e fibras foram obtidos com auxílio do software Dietwin Profissional $2008^{\circledR}$ acrescido de informações de diferentes tabelas de composição de alimentos e de rótulos de alimentos industrializados. Ressalta-se que na análise dos R24h foi inserido o consumo per capita diário de sal, açúcar e óleo de adição relatado pelos usuários.

A ingestão habitual de cada nutriente foi estimada pelo Multiple Source Method (MSM), possibilitando a estimativa da ingestão habitual de nutrientes ou alimentos ${ }^{14}$, sendo o consumo calórico avaliado de acordo com as recomendações do $\mathrm{IOM}^{13}$, a ingestão de macronutriente verificada qualitativamente, conforme os intervalos percentuais aceitáveis, com classificação em insuficiente, adequada e excessiva ${ }^{13}$, as frações lipídicas avaliadas segundo referências da Organização Mundial da Saúde - OMS ${ }^{15}$ e o colesterol e as fibras de acordo com o IOM $^{13}$.

Para avaliação do consumo alimentar utilizou-se, também, um Questionário de Frequência Alimentar (QFA) qualitativo relativo aos últimos seis meses ${ }^{16}$ e avaliou-se o fracionamento das refeições, o consumo per capita diário de açúcar e óleo de adição e o hábito de "beliscar" alimentos entre as refeições.

Realizou-se avaliação antropométrica com aferição do peso e estatura para obtenção do Índice de Massa Corporal [IMC $=$ peso $(\mathrm{kg}) /$ altura $(\mathrm{m})^{2}$ ], classificado de maneira diferenciada para adultos ${ }^{17}$ e idosos ${ }^{18}$ em que, para fins de análise, as categorias sobrepeso e obesidade foram agrupadas em uma única, denominada excesso de peso. Ainda, aferiram-se as circunferências da cintura (CC) e do quadril (CQ), sendo a primeira utilizada para avaliação do risco de complicações metabólicas associadas ao excesso de peso e, em associação com a segunda, calculado a Razão Cintura-Quadril ( $\mathrm{RCQ}=\mathrm{CC} / \mathrm{CQ})$, classificadas segundo recomendações ${ }^{19}$.

Para estimar a notificação imprecisa da ingestão energética utilizou-se o método proposto por McCrory et al. ${ }^{20}$, que considera a diferença percentual entre a ingestão energética (IE) e o gasto energético total (GET) previsto pela equação de Vinken et al. ${ }^{21}$. Os pontos de corte para determinação da notificação imprecisa foram definidos a partir do uso de \pm 2 desvios-padrão (2DP), com base no intervalo de confiança de $95 \%$, chegando-se ao valor de $46 \%$ a partir dos dados da própria amostra e metodologia adotada no estudo. Logo, definiu-se como indivíduos notificadores imprecisos aqueles cuja razão IE/GET fosse inferior a 54\% (subnotificadores) ou superior a 146\% (supernotificadores). 
Utilizou-se os programas Microsoft Access 2010 para a construção do banco de dados, Statistical Package for the Social Sciences (SPSS) for Windows versão 19.0 e Stata 11.0 como auxílio para as análises estatísticas, sendo adotado nível de significância de $5 \%$.

A análise descritiva incluiu o cálculo das distribuições de frequências e medidas de tendência central e dispersão. Aplicou-se os testes Kolmogorov-Smirnov, para verificação do padrão de distribuição das variáveis quantitativas, sendo aquelas que aderiram à distribuição normal apresentadas em média \pm desvio-padrão e, as demais, em mediana e intervalo interquartil (percentil 25 - percentil 75). Também foram realizados testes Qui-quadrado ou Exato de Fisher com a correção de Bonferroni, t de Student simples, Mann-Whitney e correlação de Spearman.

Por fim, construiu-se um modelo de regressão logística, considerando a subnotificação do consumo alimentar como variável desfecho. Utilizou-se o método backward para a construção do modelo múltiplo sendo o valor $\mathrm{p}<0,20$, obtido na análise bruta, o critério para a inclusão das variáveis explicativas, que foram definidas a partir de revisão da literatura e estudos prévios $^{3-6}$. A qualidade do ajuste foi avaliada pelo teste Hosmer \& Lemeshow e testou-se a interação entre as variáveis e o efeito de variáveis de confundimento como sexo, idade e estado nutricional.

Todos os participantes foram informados sobre os objetivos e os métodos do estudo por meio de carta de informação, e os que consentiram em participar assinaram o Termo de Consentimento Livre e Esclarecido, declarando estarem cientes dos objetivos da pesquisa. O protocolo da pesquisa no qual se insere esse projeto foi aprovado pelos Comitês de Ética em Pesquisa da Universidade Federal de Minas Gerais e da Prefeitura Municipal de Belo Horizonte.

\section{Resultados}

No período de coleta de dados foram elegíveis, segundos os critérios de inclusão, 627 indivíduos. Destes, foram excluídos aqueles que se recusaram a participar da pesquisa $(n=64)$, os que atendiam aos critérios de exclusão $(n=41)$ e aqueles usuários cujo questionário apresentava dados faltantes que impossibilitassem a classificação da notificação do consumo alimentar $(\mathrm{n}=35)$, resultando em uma amostra de 487 participantes.

Dos usuários avaliados, $82,3 \%$ eram do sexo feminino e $50,5 \%$ idosos, com mediana de idade de 60 (53 - 67) anos. Verificou-se mediana de 7 (4-11) anos de estudo, 41,5\% aposentados e renda per capita mensal de $\mathrm{R} \$ 678,00$ (R\$ 404,65 R\$ 1000,00) (Tabela 1).

Considerando o perfil de saúde, observou-se alta prevalência de HAS (52,0\%) e dislipidemias $(43,1 \%)$, sendo o uso de medicamentos referido por $76,0 \%$. A maioria das pessoas apresentava percepção de saúde boa/muito boa $(75,8 \%)$, estavam satisfeitas com seu peso corporal $(83,4 \%)$ e foram classificadas como pouco ativas segundo o nível de atividade física (92,4\%). Em relação à antropometria, aponta-se elevada prevalência de excesso de peso (36,2\%), CC elevada e muito elevada (29,4\% e $18,7 \%$, respectivamente), bem como RCQ elevada (44,2\%) (Tabela 1).

No que diz respeito aos hábitos alimentares, percebe-se baixa ingestão de frutas $(65,1 \%)$, hortaliças $(65,7 \%)$ e leite e derivados $(80,7 \%)$, além de alta prevalência de consumo de 5 ou mais refeições diárias (Tabela 2).

A avaliação do consumo alimentar a partir dos R24h apontou alta prevalência de ingestão insuficiente de calorias (49,3\%), ácidos graxos monoinsaturados $(80,9 \%)$ e de fibras $(83,8 \%)$, além da ingestão excessiva de lipídios e ácidos graxos saturados por $24,8 \%$ e $27,9 \%$, respectivamente (Tabela 3). Destaca-se que na avaliação do consumo alimentar utilizou-se o kit de medidas caseiras com 46,2\% dos participantes em pelo menos um R24h.

A prevalência de notificação imprecisa do consumo alimentar encontrada na população em estudo foi de $12,7 \%$, sendo $11,9 \%(\mathrm{n}=58)$ classificados como subnotificadores e $0,8 \%$ ( $n=$ 4) como supernotificadores. Optou-se por não avaliar os fatores associados à supernotificação devido à baixa prevalência observada, tal como efetuado por Yannakoulia et al. ${ }^{4}$.

A subnotificação foi mais prevalente entre os adultos $(\mathrm{p}=0,001)$, naqueles com dislipidemias $(\mathrm{p}=0,001)$, não satisfeitos com o peso corporal $(\mathrm{p}<0,001)$, com excesso de peso $(\mathrm{p}<0,001)$ e aqueles com CC muito elevada ( $\mathrm{p}<0,001)(\mathrm{Ta}-$ bela 1).

Ainda, não houve diferença estatisticamente significante da subnotificação segundo os hábitos alimentares, porém identificou-se entre os subnotificadores maior ingestão de proteínas ( $\mathrm{p}$ $=0,005)$. O consumo de colesterol $(\mathrm{p}<0,001)$ e fibras $(p<0,001)$ também foi menor entre esses, no entanto, quando ajustado para 1000 calorias, não houve diferença estatisticamente significante, bem como quando avaliado segundo o uso do kit de medidas caseiras (Tabela 2). 
Tabela 1. Subnotificação do consumo alimentar segundo perfil sociodemográfico e econômico, de saúde e antropométrico dos participantes. Belo Horizonte/MG, 2014.

\begin{tabular}{|c|c|c|c|c|}
\hline \multirow[t]{2}{*}{ Variável } & \multirow[b]{2}{*}{ Total } & \multicolumn{2}{|c|}{ Subnotificação } & \multirow{2}{*}{ Valor $\mathbf{p}$} \\
\hline & & Sim & Não & \\
\hline \multicolumn{5}{|l|}{ Sexo $(\%)$} \\
\hline Feminino & 82,3 & 12,3 & 87,7 & $0,688^{\mathrm{a}}$ \\
\hline Masculino & 17,7 & 10,7 & 89,3 & \\
\hline Idade $(\operatorname{anos})^{*}$ & $60,0(53,0-67,0)$ & $55,5(48,0-61,0)$ & $61(53,0-68,0)$ & $<0,001^{\mathrm{b}}$ \\
\hline \multicolumn{5}{|l|}{ Faixa etária (\%) } \\
\hline Adulto & 49,5 & 17,1 & 82,9 & $0,001^{\mathrm{a}}$ \\
\hline Idoso & 50,5 & 7,0 & 93,0 & \\
\hline Anos de estudo* & $7,0(4,0-11,0)$ & $6,0(3,0-11,0)$ & $7,0(4,0-11,0)$ & $0,470^{\mathrm{b}}$ \\
\hline Renda per capita $(\mathrm{R} \$)^{*}$ & $\begin{array}{c}678,0 \\
(404,7-1000,0)\end{array}$ & $\begin{array}{c}666,7 \\
(406,8-1000,0)\end{array}$ & $\begin{array}{c}678,0 \\
(401,9-1000,0)\end{array}$ & $0,563^{b}$ \\
\hline \multicolumn{5}{|l|}{ Profissão (\%) } \\
\hline Do lar & 24,6 & 13,4 & 86,6 & \\
\hline Aposentado & 41,5 & 9,5 & 90,5 & $0,525^{\mathrm{a}}$ \\
\hline Desempregado & 1,8 & 11,1 & 88,9 & \\
\hline Outros & 32,1 & 14,3 & 85,7 & \\
\hline \multicolumn{5}{|l|}{ Diabetes mellitus (\%) } \\
\hline Não & 83,8 & 10,9 & 89,1 & $0,166^{\mathrm{a}}$ \\
\hline Sim & 16,2 & 16,5 & 83,5 & \\
\hline \multicolumn{5}{|l|}{ Hipertensão arterial (\%) } \\
\hline Não & 48,0 & 9,5 & 90,5 & $0,108^{\mathrm{a}}$ \\
\hline $\operatorname{Sim}$ & 52,0 & 14,3 & 85,7 & \\
\hline \multicolumn{5}{|l|}{ Dislipidemias (\%) } \\
\hline Não & 56,9 & 7,9 & 92,1 & $0,001^{\mathrm{a}}$ \\
\hline Sim & 43,1 & 17,6 & 82,4 & \\
\hline \multicolumn{5}{|l|}{ Morbidade psiquiátrica (\%) } \\
\hline Não & 87,1 & 11,4 & 88,6 & $0,312^{\mathrm{a}}$ \\
\hline Sim & 12,9 & 15,9 & 84,1 & \\
\hline \multicolumn{5}{|l|}{ Uso de medicamentos (\%) } \\
\hline Não & 24,0 & 9,6 & 90,4 & $0,356^{\mathrm{a}}$ \\
\hline $\operatorname{Sim}$ & 76,0 & 12,8 & 87,2 & \\
\hline \multicolumn{5}{|l|}{ Tabagismo (\%) } \\
\hline Não & 94,7 & 12,0 & 88,0 & $0,940^{\mathrm{a}}$ \\
\hline Sim & 5,3 & 11,5 & 88,5 & \\
\hline \multicolumn{5}{|l|}{ Percepção de saúde (\%) } \\
\hline Muito ruim - Ruim & 1,6 & 37,5 & 62,5 & \\
\hline Razoável & 22,6 & 12,7 & 87,3 & $0,075^{\mathrm{a}}$ \\
\hline Bom - Muito Bom & 75,8 & 11,2 & 88,8 & \\
\hline \multicolumn{5}{|c|}{ Satisfação com o peso corporal (\%) } \\
\hline Não & 16,6 & 24,7 & 75,3 & $<0,001^{\mathrm{a}}$ \\
\hline Sim & 83,4 & 9,5 & 90,5 & \\
\hline \multicolumn{5}{|c|}{ Orientação para mudança de hábitos alimentares (\%) } \\
\hline Não & 51,2 & 12,1 & 87,9 & $0,938^{\mathrm{a}}$ \\
\hline Sim & 48,8 & 11,9 & 88,1 & \\
\hline
\end{tabular}


Tabela 1. continuação

\begin{tabular}{|c|c|c|c|c|}
\hline \multirow[t]{2}{*}{ Variável } & \multirow[b]{2}{*}{ Total } & \multicolumn{2}{|c|}{ Subnotificação } & \multirow{2}{*}{ Valor p } \\
\hline & & Sim & Não & \\
\hline \multicolumn{5}{|l|}{ Nível de atividade física (\%) } \\
\hline Sedentário & 7,6 & 13,5 & 86,5 & $0,769^{\mathrm{a}}$ \\
\hline Pouco ativo & 92,4 & 11,9 & 88,1 & \\
\hline \multicolumn{5}{|l|}{ Índice de Massa Corporal (\%) } \\
\hline Baixo peso & 9,0 & 2,4 & 97,6 & \\
\hline Eutrofia & 54,8 & 7,5 & 92,5 & $<0,001^{\mathrm{c}}$ \\
\hline Excesso de peso & 36,2 & 21,1 & 78,9 & \\
\hline \multicolumn{5}{|l|}{ Circunferência da cintura (\%) } \\
\hline Normal & 51,9 & 7,7 & 92,3 & \\
\hline Elevada & 29,4 & 9,1 & 90,9 & $<0,001^{\mathrm{d}}$ \\
\hline Muito elevada & 18,7 & 28,6 & 71,4 & \\
\hline \multicolumn{5}{|l|}{ Razão Cintura-Quadril (\%) } \\
\hline Normal & 55,8 & 11,2 & 88,8 & $0,526^{\mathrm{a}}$ \\
\hline Elevada & 44,2 & 13,1 & 86,9 & \\
\hline
\end{tabular}

Nota: ${ }^{*}$ Resultados apresentados como mediana (p25-75). a Teste Qui-quadrado ou exato de Fisher. ${ }^{\mathrm{b}}$ Teste Mann-Whitney.

${ }^{\mathrm{c}}$ Diferença estatística entre as categorias excesso de peso e baixo peso $(\mathrm{p}=0,004)$ e excesso de peso e eutrofia $(\mathrm{p}<0,001)$.

${ }^{\mathrm{d}}$ Diferença estatística entre as categorias muito elevada e normal $(\mathrm{p}<0,001)$ e muito elevada e elevada $(\mathrm{p}<0,001)$.

Verificou-se correlação direta entre a razão IE/GET com a idade $(\mathrm{r}=0,240 ; \mathrm{p}<0,001)$, renda per capita $(\mathrm{r}=0,166 ; \mathrm{p}<0,001)$ e consumo per capita diário de açúcar $(\mathrm{r}=0,240 ; \mathrm{p}<0,001)$ e de óleo de adição $(r=0,262 ; \mathrm{p}<0,001)$.

Por fim, verificou-se, por meio da análise multivariada, que a subnotificação do consumo alimentar foi mais prevalente entre os participantes que apresentavam dislipidemias $(\mathrm{RP}=3,39$; IC95\%: 1,73;6,67) e circunferência da cintura muito elevada ( $R P=6,58$; IC95\%: 2,95;14,66), e menos prevalente entre os adultos $(\mathrm{RP}=0,22$; IC95\%: 0,11;0,46), aqueles satisfeitos com o peso corporal $(\mathrm{RP}=0,42$; IC95\%: 0,19;0,89) e que apresentavam fracionamento da dieta igual ou superior a cinco refeições diárias $(\mathrm{RP}=0,49$; IC95\%: 0,25;0,98) (Tabela 4).

\section{Discussão}

O presente estudo apontou baixa prevalência de notificação imprecisa da ingestão energética e evidenciou associação da subnotificação com a faixa etária, comorbidades, satisfação com o peso corporal, CC e fracionamento da dieta. Houve ainda maior tendência a subnotificação com o incremento da idade, renda e consumo per capita diário de açúcar e óleo de adição.
A prevalência de supernotificação encontra$\mathrm{da}(0,8 \%)$ foi semelhante à de outros trabalhos, cujos valores variaram de $0,0 \%$ a $7,9 \% \%^{3,4,11,22}$, confirmando que o principal problema em estudos que abrangem a avaliação dietética é a subnotificação ${ }^{11}$.

A prevalência dessa, por sua vez, observada neste trabalho $(11,9 \%)$, foi também similar a outros que utilizaram a mesma metodologia para sua detecção e para avaliação do consumo alimentar, como o de Avelino et al..$^{23}$, que apontou prevalência de $15,1 \%$, e o de McCrory et al. ${ }^{20}$, que encontrou $11 \%$ de notificação imprecisa (não houve especificação de sub ou supernotificação, porém destacou-se que a grande maioria desse valor correspondia à subnotificação). No entanto, foi inferior à encontrada em outros trabalhos ${ }^{24-26}$.

Como já apontado na literatura, a notificação imprecisa é desigualmente distribuída na população ${ }^{3}$ e torna difícil e limitada a realização de comparações entre essas prevalências nos diversos estudos pela diferença entre os métodos utilizados para sua detecção ${ }^{24,25}$ e para avaliação dietética ${ }^{27,28}$.

Destaca-se que, no presente estudo, a baixa prevalência se deve, provavelmente, ao rigor metodológico adotado durante a coleta de dados do consumo alimentar, o que possibilitou melhor 
Tabela 2. Subnotificação do consumo alimentar segundo hábitos e consumo alimentar dos participantes. Belo Horizonte/MG, 2014.

\begin{tabular}{|c|c|c|c|c|}
\hline \multirow[t]{2}{*}{ Variável } & \multirow[b]{2}{*}{ Total } & \multicolumn{2}{|c|}{ Subnotificação } & \multirow[t]{2}{*}{ Valor $\mathrm{p}$} \\
\hline & & Sim & Não & \\
\hline \multicolumn{5}{|l|}{ Fracionamento da dieta (\%) } \\
\hline$<5$ refeições diárias & 26,5 & 15,6 & 84,4 & $0,142^{\mathrm{a}}$ \\
\hline$\geq 5$ refeições diárias & 73,5 & 10,7 & 89,3 & \\
\hline \multicolumn{5}{|c|}{ Hábito de beliscar alimentos entre as refeições (\%) } \\
\hline Não & 61,4 & 12,8 & 87,2 & $0,524^{\mathrm{a}}$ \\
\hline Sim & 38,6 & 10,8 & 89,2 & \\
\hline \multicolumn{5}{|l|}{ Consumo de frutas (\%) } \\
\hline$<3$ porções diárias & 65,1 & 12,4 & 87,6 & $0,704^{\mathrm{a}}$ \\
\hline$\geq 3$ porções diárias & 34,9 & 11,2 & 88,8 & \\
\hline \multicolumn{5}{|l|}{ Consumo de hortaliças (\%) } \\
\hline$<3$ porções diárias & 65,7 & 13,3 & 86,7 & $0,233^{\mathrm{a}}$ \\
\hline$\geq 3$ porções diárias & 34,3 & 9,6 & 90,4 & \\
\hline \multicolumn{5}{|l|}{ Consumo de leite e derivados (\%) } \\
\hline$<3$ porções diárias & 80,7 & 12,6 & 87,4 & $0,442^{\mathrm{a}}$ \\
\hline$\geq 3$ porções diárias & 19,3 & 9,7 & 90,3 & \\
\hline \multicolumn{5}{|l|}{ Consumo de embutidos (\%) } \\
\hline$\geq 2$ vezes mensais & 47,4 & 10,5 & 89,5 & $0,343^{\mathrm{a}}$ \\
\hline$<2$ vezes mensais & 52,6 & 13,3 & 86,7 & \\
\hline \multicolumn{5}{|l|}{ Consumo de doces (\%) } \\
\hline$>2$ vezes semanais & 22,3 & 9,4 & 90,6 & $0,347^{\mathrm{a}}$ \\
\hline$\leq 2$ vezes semanais & 77,7 & 12,8 & 87,2 & \\
\hline \multicolumn{5}{|l|}{ Consumo de frituras (\%) } \\
\hline$>2$ vezes semanais & 14,4 & 10,1 & 89,9 & $0,607^{\mathrm{a}}$ \\
\hline$\leq 2$ vezes semanais & 85,6 & 12,3 & 87,7 & \\
\hline \multicolumn{5}{|c|}{$\begin{array}{l}\text { Consumo de salgados, sanduíches e salgadinhos } \\
\text { tipo "chips" (\%) }\end{array}$} \\
\hline$\geq 2$ vezes mensais & 25,1 & 16,8 & 83,2 & $0,064^{\mathrm{a}}$ \\
\hline$<2$ vezes mensais & 74,9 & 10,4 & 89,6 & \\
\hline \multicolumn{5}{|l|}{ Uso do kit de medidas caseiras no R24h (\%) } \\
\hline Não & 53,8 & 11,9 & 88,1 & $0,950^{\mathrm{a}}$ \\
\hline Sim & 46,2 & 12,1 & 87,9 & \\
\hline Ingestão de calorias $(\mathrm{kcal})^{*}$ & $\begin{array}{c}1598,7 \\
(1344,9-1847,3)\end{array}$ & $\begin{array}{c}1066,9 \\
(896,4-1240,5)\end{array}$ & $\begin{array}{c}1649,3 \\
(1454,3-1909,8)\end{array}$ & $<0,001^{\mathrm{b}}$ \\
\hline Ingestão de carboidratos (\% IE) ${ }^{* *}$ & $56,6 \pm 7,5$ & $54,8 \pm 8,7$ & $54,4 \pm 7,3$ & $0,704^{\mathrm{c}}$ \\
\hline Ingestão de proteínas (\% IE) ${ }^{* *}$ & $14,7 \pm 3,2$ & $15,8 \pm 3,4$ & $14,5 \pm 3,2$ & $0,005^{\mathrm{c}}$ \\
\hline Ingestão de lipídios (\%IE) ${ }^{* *}$ & $30,8 \pm 6,7$ & $30,6 \pm 6,9$ & $30,9 \pm 6,6$ & $0,759^{c}$ \\
\hline Ingestão de AG saturados (\% IE) ${ }^{* *}$ & $8,8 \pm 2,2$ & $8,9 \pm 2,1$ & $8,8 \pm 2,1$ & $0,792^{\mathrm{c}}$ \\
\hline Ingestão de AG monoinsaturados (\% IE) ${ }^{* *}$ & $8,3 \pm 1,9$ & $8,2 \pm 2,1$ & $8,3 \pm 1,9$ & $0,803^{\mathrm{c}}$ \\
\hline Ingestão de AG poliinsaturados (\% IE) ${ }^{*}$ & $8,5(6,7-11,0)$ & $8,4(6,4-11,3)$ & $8,5(6,8-11,1)$ & $0,863^{\mathrm{b}}$ \\
\hline Ingestão de colesterol $(\mathrm{mg})^{*}$ & $\begin{array}{c}174,8 \\
(131,9-213,9)\end{array}$ & $\begin{array}{c}127,1 \\
(96,7-168,9)\end{array}$ & $\begin{array}{c}178,9 \\
(138,3-219,2)\end{array}$ & $<0,001^{\mathrm{b}}$ \\
\hline Ingestão de colesterol $(\mathrm{mg} / 1000 \mathrm{cal})^{* *}$ & $110,4 \pm 37,0$ & $119,4 \pm 48,0$ & $109,4 \pm 35,2$ & $0,130^{c}$ \\
\hline Ingestão de fibras $(\mathrm{g})^{*}$ & $16,3(12,6-20,9)$ & $10,9(9,2-14,2)$ & $16,9(13,6-21,8)$ & $<0,001^{\mathrm{b}}$ \\
\hline Ingestão de fibras $(\mathrm{g} / 1000 \mathrm{cal})^{*}$ & $10,3(8,3-12,7)$ & $11,1(7,9-13,2)$ & $10,3(8,3-12,7)$ & $0,534^{\mathrm{b}}$ \\
\hline
\end{tabular}

Nota: " Resultados apresentados como Mediana (p25-p75). ${ }^{* *}$ Resultados apresentados como Média \pm Desvio-padrão. ${ }^{a}$ Teste Quiquadrado ou exato de Fisher. ${ }^{\mathrm{b}}$ Teste Mann-Whitney. ${ }^{\mathrm{c}}$ Teste $\mathrm{t}$ de Student. AG: Ácidos Graxos; g: gramas; IE: Ingestão Energética; mg: miligramas; g: gramas; ml: mililitros. 
Tabela 3. Adequação do consumo de calorias e nutrientes da amostra. Belo Horizonte/MG, 2014.

\begin{tabular}{|c|c|c|}
\hline Variável & $\mathbf{n}$ & Valores \\
\hline \multicolumn{3}{|l|}{ Calorias (\%) } \\
\hline Insuficiente & 240 & 49,3 \\
\hline Adequado & 210 & 43,1 \\
\hline Excessivo & 37 & 7,6 \\
\hline \multicolumn{3}{|l|}{ Carboidratos (\%) } \\
\hline Insuficiente & 55 & 11,3 \\
\hline Adequado & 404 & 83,0 \\
\hline Excessivo & 28 & 5,7 \\
\hline \multicolumn{3}{|l|}{ Proteínas (\%) } \\
\hline Insuficiente & 26 & 5,3 \\
\hline Adequado & 461 & 94,7 \\
\hline \multicolumn{3}{|l|}{ Lipídios (\%) } \\
\hline Insuficiente & 17 & 3,5 \\
\hline Adequado & 349 & 71,7 \\
\hline Excessivo & 121 & 24,8 \\
\hline \multicolumn{3}{|c|}{ Ácidos graxos saturados (\%) } \\
\hline Adequado & 351 & 72,1 \\
\hline Excessivo & 136 & 27,9 \\
\hline \multicolumn{3}{|c|}{ Ácidos graxos monoinsaturados (\%) } \\
\hline Insuficiente & 394 & 80,9 \\
\hline Adequado & 93 & 19,1 \\
\hline \multicolumn{3}{|c|}{ Ácidos graxos poliinsaturados (\%) } \\
\hline Insuficiente & 87 & 17,8 \\
\hline Adequado & 276 & 56,7 \\
\hline Excessivo & 124 & 25,5 \\
\hline \multicolumn{3}{|l|}{ Colesterol (\%) } \\
\hline Adequado & 453 & 93,0 \\
\hline Excessivo & 34 & 7,0 \\
\hline \multicolumn{3}{|l|}{ Fibras (\%) } \\
\hline Insuficiente & 408 & 83,8 \\
\hline Adequado & 79 & 16,2 \\
\hline
\end{tabular}

estimativa da real ingestão energética dos indivíduos. Ainda, por se tratar de usuários que estão inseridos em um Serviço de Promoção da Saúde, provavelmente há maior preocupação com a saúde e, consequentemente, com a alimentação, aumentando a tendência do relato de forma mais precisa em busca de um retorno quanto à adequação daquele consumo.

No que diz respeito à relação da subnotificação com a idade, alguns estudos apontaram maior prevalência entre as pessoas mais velhas ${ }^{22,24,25}$ contradizendo os resultados aqui evidenciados. No entanto, relação similar foi encontrada por Yannakoulia et al. $^{4}$, que detectaram menor razão IE/TMB (usada para detecção da tendência
Tabela 4. Modelo final da Regressão Logística de variáveis associadas à subnotificação do consumo alimentar. Belo Horizonte/MG, 2014.

\begin{tabular}{lccc}
\hline \multicolumn{1}{c}{ Variáveis } & RP & IC95\% & Valor p \\
\hline Faixa etária & & & \\
Adulto & 1,00 & - & \\
Idoso & 0,22 & 0,$11 ; 0,46$ & $<0,001$ \\
Dislipidemias & & & \\
Não & 1,00 & - & \\
Sim & 3,39 & 1,$73 ; 6,67$ & $<0,001$ \\
Satisfação com o peso & & & \\
corporal & & & \\
Não & 1,00 & - & \\
Sim & 0,42 & 0,$19 ; 0,89$ & 0,024 \\
Circunferência da cintura & & & \\
Normal & 1,00 & - & \\
Elevada & 1,48 & 0,$66 ; 3,33$ & 0,345 \\
Muito elevada & 6,58 & 2,$95 ; 14,66$ & $<0,001$ \\
Fracionamento adequado & & & \\
da dieta & & & \\
Não & 1,00 & - & \\
Sim & 0,49 & 0,$25 ; 0,98$ & 0,042 \\
\hline
\end{tabular}

Nota: RP: Razão de Prevalência; IC: Intervalo de Confiança.

Teste de qualidade do ajuste: $\mathrm{p}=0,9847$.

de subnotificação) entre pessoas mais jovens, justificando-se pela melhor consciência dessas sobre saúde e dieta. Assim, haveria uma maior tendência em não notificar o consumo alimentar verdadeiro, mas sim o que seria mais adequado segundo padrões de uma alimentação saudável.

Já a maior prevalência de subnotificação entre as pessoas que relataram presença de dislipidemias, também detectada por outros autores, pode indicar maior abrangência de informações sobre alimentação e saúde recebidas pelas pessoas doentes, favorecendo o relato mais aproximado das orientações recebidas ${ }^{4}$. Ademais, por ser uma doença revertida com a mudança da alimentação na sua fase aguda, propicia tentativas de melhorias dietéticas a fim de contribuir para o controle da situação.

Além da associação com condições de saúde, a subnotificação também pode se associar a aspectos psicológicos, tal como evidenciado com a insatisfação com o peso corporal. Esse parâmetro, comumente está atrelado ao descontentamento com a imagem corporal e ao desejo de ajuste social, que favorecem uma resposta mais aceitável socialmente ${ }^{23,27}$. Rasmussem et al. ${ }^{11}$ ain- 
da encontraram associação com a tentativa de comer de forma mais saudável e a preocupação com o peso corporal.

Associado a esse fator encontra-se a relação com o excesso de peso, que é bem relatada pela literatura ${ }^{3,4,11,22-26}$ em virtude da comum insatisfação corporal evidenciada nestes $\operatorname{casos}^{22,27}$. No presente estudo observou-se relação independente entre subnotificação e a classificação de CC muito elevada, que também é um indicativo de excesso de peso, principalmente no que diz respeito ao excesso de gordura abdominal ${ }^{29}$.

Em relação aos hábitos alimentares, a maior prevalência de subnotificação entre aqueles com fracionamento inadequado da dieta também foi apontada na revisão bibliográfica realizada por Maurer et al. ${ }^{30}$, que indicou maior omissão de lanches entre as refeições nos subnotificadores e, consequentemente, menor número diário de refeições.

Destaca-se como limitações deste trabalho o delineamento transversal; e o uso de equações preditivas para detecção da notificação imprecisa do consumo alimentar. No entanto, o uso de técnicas padrão-ouro, como a água duplamente marcada, torna-se inviável em trabalhos que utili- zam grandes amostras devido seu custo e complexidade; e a impossibilidade de avaliação daquelas pessoas que estão em atual tentativa de mudança de peso, limitação essa inerente ao próprio método para detecção da notificação imprecisa.

Cabe salientar a inovação da realização desta pesquisa no âmbito da Atenção Primária à Saúde. Por se tratar de usuários de um serviço onde são realizadas atividades de intervenção que buscam melhora dos hábitos alimentares para recuperação ou prevenção da saúde, faz-se necessário o conhecimento de forma mais fidedigna possível do consumo alimentar deste público, a fim de possibilitar o atendimento das suas especificidades.

A partir desses resultados e diante da importância dessa avaliação para a melhor qualidade dos dados de avaliação do consumo alimentar, ressalta-se a necessidade do uso de estratégias para reduzir sua prevalência, seja com a utilização de técnicas alternativas para coleta de dados dietéticos ou de programas de orientação aos indivíduos sobre a importância e a necessidade do relato preciso, principalmente aqueles que apresentam maior tendência a notificar de forma incorreta.

\section{Colaboradores}

CH Machado e LC Santos trabalharam na concepção e redação do artigo e aprovação da versão a ser publicada e ACS Lopes na redação do artigo e aprovação da versão a ser publicada.

\section{Referências}

1. Biltoft-Jensen A, Matthiessen J, Rasmussen LB, Fagt S, Groth MV, Hels O. Validation of the Danish 7-day pre-coded food diary among adults: energy intake v. energy expenditure and recording length. $\mathrm{Br} J \mathrm{Nutr}$ 2009; 102(12):1838-1846.

2. Holanda LB, Barros Filho AA. Métodos aplicados em inquéritos alimentares. Rev Paul Pediatr 2006; 24(1):62-70.

3. Bazelmans C, Matthys C, De Henauw S, Dramaix M, Kornitzer M, De Backer G, Levêque A. Predictors of misreporting in na elderly population: the 'Quality of life after 65' study. Public Health Nutr 2007; 10(2):185191.

4. Yannakoulia M, Panagiotakos DB, Pitsavos C, Bathrellou E, Chrysohoou C, Skoumas Y, Stefanadis C. Low energy reporting related to lifestyle, clinical, and psychosocial factors in a randomly selected population sample of greek adults: the ATTICA study. J Am College Nutr 2007; 26(4):327-333.

5. Bazanelli AP, Kamimura MA, Vasselai P, Draibe AS, Cuppari L. Underreporting of energy intake in peritoneal dialysis patients. J Renal Nutr 2009; 20(4):263-269. 
6. Poslusna K, Ruprich J, de Vries JH, Jakubikova M, van't Veer P. Misreporting of energy and micronutrient intake estimated by food records and 24 hour recalls, control and adjustment methods in practice. $\mathrm{Br} \mathrm{J} \mathrm{Nutr}$ 2009; 101(Supl. 2):S73-86.

7. Scagliusi FB, Lancha Júnior AH. Estudo do gasto energético por meio da água duplamente marcada: fundamentos, utilização e aplicações. Rev nutr 2005; 18(4):541-551.

8. Brasil. Ministério da Saúde (MS). Portaria n ${ }^{\circ} 2.681$, de 7 de novembro de 2013. Redefine o Programa Academia da Saúde no âmbito do Sistema Único de Saúde (SUS). Diário Oficial da União 2013; 8 nov.

9. Dias MAS, Lopes ACS, Bicalho K, Mourão M, Alves MN, Evangelista PA, Rodrigues RCLC, Guimarães VR. Promoção à saúde e articulação intersetorial. In: Magalhães Júnior HM, organizador. Desafios e inovações da gestão do SUS em Belo Horizonte: a experiência de 2003 a 2008. Belo Horizonte: Mazza Edições; 2010. p. 63-124.

10. Browner WS, Newman TB, Cummings SR, Hulley SB. Estimando o tamanho de amostra e o poder estatístico: pontos básicos. In: Hulley SB, Cummings SR, Browner WS, Grady DG, Newman TB, organizadores. Designing clinical research. Baltimore: Lippincott Williams \& Wiekins; 2001.

11. Rasmussen LB, Matthiessen J, Biltoft-Jensen A, Tetens I. Characteristics of misreporters of dietary intake and physical activity. Public Health Nutr 2007; 10(3):230237.

12. Andreoli SB, Mari JJ, Blay SL, Almeida-Filho N, Coutinho E, França J, Fernandes JG, Busnello ED. Estrutura fatorial do questionário de morbidade psiquiátrica de adultos aplicado em amostras populacionais de cidades brasileiras. Rev Saude Publica 1994; 28(4):249-260.

13. Otten JJ, Hellwig JP, Meyers LD, editors. Dietary Reference Intakes: The Essential Guide to Nutrient Requirements. Washington: The National Academies Press; 2006.

14. Harttiq U, Haubrock J, Knuppel S, Boeing H, Efcoval Consortium. The MSM program: web-based statistics package for estimating usual dietary intake using the Multiple Source Method. Eur J Clin Nutr 2011; 65(Supl. 1):87-91.

15. World Health Organization (WHO). Interim Summary of Conclusions and Dietary Recommendations on Total Fat \& Fatty Acids. Geneva: From the Joint World Health Organization/Food and Agriculture Organization. Expert Consultation on Fats and Fatty Acids in Human Nutrition; 2008.

16. Lopes ACS, Ferreira AD, Santos LC. Atendimento nutricional na Atenção Primária à Saúde: proposição de protocolos. Nutrição em pauta 2010; 18(101):1-6.

17. World Health Organization (WHO). Physical Status: the use and interpretation of anthropometry. Geneva: WHO; 1995.
18. Nutrition Screening Initiative. Nutrition interventions manual for professionals caring for older Americans: project of the American Academy of Family Physicians. Washington: Nutrition Screening Initiative; 1994.

19. World Health Organization (WHO). Waist circumference and waist-hip ratio: report of a WHO expert consultation. Geneva: WHO; 2011.

20. McCrory MA, Hajduk CL, Roberts SB. Procedures for screening out inaccurate reports of dietary energy intake. Public Health Nutr 2002; 5(6A):873-882.

21. Vinken AG, Bathalon GP, Sawaya AL, Dallal GE, Tucker KL, Roberts SB. Equations for predicting the energy requirements of healthy adults aged 18-81 y. Am J Clin Nutr 1999; 69(5):920-926.

22. Gomes AA, Leão LSCS. Prevalência de sub-relato e super-relato de ingestão energética em população ambulatorial do Rio de Janeiro, Brasil. Cad Saúde Coletiva 2011; 19(2):197-202.

23. Avelino GF, Previdelli NA, Castro MA, Marchioni DML, Fisberg RM. Sub-relato da ingestão energética e fatores associados em estudo de base populacional. $\mathrm{Cad}$ Saude Publica 2014; 30(3):663-668.

24. Mirmiran P, Esmaillzadeh A, Azizi F. Under-reporting of energy intake affects estimates of nutriente intakes. Asia Pac j clin nutr 2006; 15(4):459-464.

25. Azizi F, Esmaillzadeh A, Mirmiran P. Correlates of under- and over-reporting of energy intake in Tehranians: body mass index and lifestyle-related factores. Asia Pac j clin nutr 2005; 14(1):54-59.

26. Mendez MA, Wynter S, Wilks R, Forrester T. Underand overreporting of energy is related to obesity, lifestyle factors and food group intakes in Jamaican adults. Public health nutr 2004; 7(1):9-19.

27. Luhrmann PM, Herbert BM, Neuhauser-Berthold M. Underreporting of energy intake in an elderly german population. Nutrition 2001; 17(11-12):912-916.

28. Scagliusi FB, Ferrioli E, Pfrimer K, Laureano C, Cunha CS, Gualano B, Lourenço BH, Lancha AH Jr. Underreporting of energy intake in Brazilian women varies according to dietary assessment: a cross-sectional study using doubly labeled water. J Am Diet Assoc 2008; 108(12):2031-2040.

29. World Health Organization (WHO). Diet, nutrition and the prevention of chronic diseases. Geneva: Report of a Joint World Health Organization/Food and Agriculture Organization; 2003.

30. Maurer J, Taren DL, Teixeira PJ, Thomson CA, Lohman TG, Going SB, Houtkooper LB. The psychosocial and behavioral characteristics related to energy misreporting. Nutr rev 2006; 64(2):53-66.

Artigo apresentado em 14/10/2015

Aprovado em 17/12/2015

Versão final apresentada em 19/12/2015 